\title{
Guest Editorial: MobiHealth 2014, IEEE HealthCom 2014 and IEEE BHI 2014
}

\author{
The patient always at the center: from patient monitoring, m-health solutions, \\ networking and security to collaborative environments, multiple source data \\ analysis and predictive modelling
}

$\mathrm{I}^{\mathrm{N}}$ $\mathrm{N}$ this special section, we gathered papers from three well known conferences organized in 2014: EAI Mobihealth, IEEE HealthCom and IEEE Biomedical and Health Informatics. EAI Mobihealth is an annually organized conference, which started in 2010 , to address the demands of the rapidly evolving disciplines of wireless communications, mobile computing and sensing technologies in healthcare. The IEEE-Healthcom is held every year since 1999 in different countries in Asia, Europe and in America. It aims at bringing together interested parties working in the field of healthcare to exchange ideas, discuss innovative and emerging solutions, and develop collaborations. The IEEE Biomedical Health Informatics (BHI) Conference started in 2013 and is organized every year providing the forum to showcase enabling technologies of computing, devices, imaging, sensors, and systems that optimize the acquisition, transmission, processing, storage, retrieval, visualization, and analysis of medical data.

The aim of this special section is to present an overview of recent advances in sensing technologies, monitoring of patients, security and privacy of data transfer, provision of collaborative environments, data gathering and analysis from various sources and predictive models, which all finally target the best strategy for patient monitoring and treatment. Eight papers are briefly presented to demonstrate that in the last decade applications, tools and models have been developed to assist in that direction and improve treatment outcomes, targeting: (a) the patient who is always in the center, (b) other actors who are involved in the healthcare system today, and (c) the availability and continuously increasing of medical data which can be collected by sensors based on m-health solutions or in various clinical settings and need further analysis and can be employed in the development of predictive computational models.

Rapid advancements in wearable sensors and smart devices are creating a pervasive wireless environment that can address a wide range of major health-related challenges, such as the diagnosis of prevalent medical conditions, monitoring of patients with chronic diseases, support of ageing population and computer-assisted rehabilitation therapies. Through the increased sensing capability and the potential of continuous measurements, data collection may be transported from the clinical setting to the free living environment, providing a wealth of acquired information. This transition from snapshot measurements of physiological parameters to continuous monitoring enables the clinicians to provide guidance on a daily basis, transforming the shape of health decision-making and disease management.

A. R. Fekr et al., in the paper entitled "Respiration disorders classification with informative features for m-health applications", introduce a system based on the use of motion sensors to detect the changes in the anterior-posterior diameter of the chest wall during breathing function. The authors combine novel feature extraction techniques along with wellknown classification schemes to distinguish breathing patterns and investigate the factors that affect the classification performance. They demonstrated accuracy rates greater than 98\% towards the discrimination between healthy people and patients with breath problems.

In the paper "Body sensor network-based spasticity detection", B. Misgeld et al. present their work towards spasticity detection, introducing an electromyography bodyworn sensor node employed in human locomotion. Furthermore, they propose a novel algorithm that focuses on the correlation analysis of antagonistically working muscles, aiming to detect an index that quantifies their co-activation. The results obtained through the analysis of the measurements of the sensor network reveal promising performance in the detection of co-active muscles associated with spasticity, driving therefore to new applications towards the reduction of the effect of spasticity.

P. C. Liang et al. focus on the monitoring of daily living of the elderly aiming to improve the accuracy of indoors location tracking systems. In their article, "Smartphone-based real-time indoor location tracking with one-meter precision", they demonstrate a localization system that can identify a patient's real-time location with high precision. More specifically, through a sensor fusion approach, developing a detector using a smart phone and a radio-based localization subsystem, they reported a quite low estimation error of 0.47 meters, enabling the adaptation of the proposed approach into a home environment.

Communications and Networking are considered as being enabling technologies for telemedicine applications in remote locations, but also for continuous health monitoring out of hospital, at home, and during sports, leisure or professional activities. Wireless Medical Sensor Networks (WMSN) have recently attracted much interest in our e-Health research community because of the wide range of applications in healthcare. But in the health domain, there is an increased issue with accessibility, integrity and privacy of data from various distributed sources of information and Medical 
devices. Even more in emergency situations, a doctor or nurse needs to access data immediately, since the loss or nonaccessibility to patient data can have dramatic consequences. In such cases, the availability of data is more important than any security concern in emergency situations.

In the paper "BTG-AC: Break-The-Glass Access Control Model for Medical Data in Wireless Sensor Networks', H-A. Maw et al., revisited the "Break-The-Glass Role-Based Access Control" (BTG-RBAC) to propose the Break-TheGlass-Access-Control (BTG-AC) model, which contributes to address with flexibility the issue with medical data availability in WMSNs and to detect the security policy violations from both authorized and unauthorized users. Their evaluation shows that the concepts of BTG, prevention and detection mechanism, and obligation provides more flexible access than other current access control models in WSNs.

The democratization of wearable sensing technologies with smartphones based applications, have greatly enhanced humans' ability of capturing and recording their "lifelong" made from various data of their daily life (steps, sleeping time, food intake, volume in communication, etc.). The impact on individual human self-behavior was formerly addressed, but in "Towards Health Exercise Behavior change for Teams Using Life-logging", Y. Nishiyama et al., have investigated the changes in the behavior of teams. They proposed and evaluated six different types of lifelong sharing model among team members for their exercise promotion, leveraging concepts of "competition" and "collaboration." According to their experiments with a mobile web application on smartphones for exercise promotion and extensive study among 64 total users for 3 weeks, the "competition" model was the most effective performance for competitive teams.

Some voice disorders, like dysphonia, frequently occur in some occupational voice users due to the extraordinary vocal load placed on the laryngeal system while exercising their occupation. Ambulatory monitoring of voice using surface electromyography (sEMG) of the extralaryngeal muscles can be an innovative approach to understand vocal hyperfunction and ultimately to monitor it in heavy voice users.

In "Detection of Vocal Dysfunctions using Complex sEMG Patterns", N. R. Smith et al., at University Missouri, USA, proposed an ambulatory EMG device to log data from multiple differential sEMG sensor channels connected to the anterior neck of the subject. A hierarchical algorithm was developed for source signal separation and when tested on 4 subjects it achieved a classification accuracy over $90 \%$ for six gestures. Furthermore, the accuracy for detection of intrasubject vocal dysfunction was $97 \%$. This demonstrates the potential of ambulatory sEMG signals collection at the anterior neck for the early detection of vocal disorders.

In silico predictive modeling can assist the treatment planning process and support the physician to adapt therapy and patient treatment. Those models are often based on features and measurements collected from different sites and in this context maximizing the consistency of data improves the outcome of statistical analysis and prediction.

Belfatto et. al. in their paper "Adaptive Mathematical Model of Tumor Response to Radiotherapy Based on CBCT data" presented a mathematical macroscopic model for the evolution of active, inactive and necrotic tumor volumes, combining a personalized approach, but also an adaptation strategy based on a group-specific optimization to assist treatment. The model parameter learning was based on cone CT imaging in 13 patients, while the adaptation testing was performed on three other patients.

Auzias et. al. in their paper "On the Influence of Confounding Factors in Multi - site Brain Morphology Studies of Developmental Pathologies: Application to Autism Spectrum Disorder" presented a study which is based on the pooling of images from different sources (MR scanners) to enhance the statistical power of analysis and identify the factors which contribute the most to cortical thickness variations, including: (i) the scanner and the disease factors and (ii) the scanner, the age and their interaction. They investigated which cortical areas suffer from age-related thinning and which areas are differentially affected by age.

The promising results, mainly related to the increased accuracy in predicting and detecting health disorders as well as to the precise and accurate identification of a patient's location in his living environment, may lead to significant progress in monitoring and management of the corresponding diseases. Data can be analyzed to support diagnostic decisions and support the clinicians' treatment decisions, but their collection is mainly dominated by security of communications and issues related to data privacy. Data can be collected by various sources, but the quality of analysis, is affected by the quality of the source as well as the consistency of measurements. Harmonising cohorts, data sources and datasets will improve the quality of the decision outcome. Finally, predictions can be performed using predictive computational models, having parameters which must be tuned to avoid limitations such as suboptimal patient stratification. 
Guest Editors

METIN AKAY

University of Houston, Houston, USA

GOUENOU COATRIEUX

Telecom Bretagne, Brest, France

YANG HAO

Queen Mary University of London, UK

DIMITRIOS I. FOTIADIS

University of Ioannina, and IMBB/BRI - FORTH, Ioannina, Greece

ANDREW LAINE

Columbia University, New York, USA

BENNY LO

Imperial College London, UK
KONSTANTINA S. NIKITA

School of Electrical and Computer Engineering, National Technical University of Athens, Athens, Greece

NORBERT NOURY

University of Lyon, Lyon, France

JOEL RODRIGUES

Dept. of Informatics, University of Beira Interior, Beira, Portugal

MAY D. WANG

Georgia Tech and Emory University, Atlanta, USA 